\title{
Electronic Forecasting of Women's Jumping Events using Neural Networks
}

Fares Ghanem Ahmed

College of Dentistry Medicine,

University of Mosul, Mosul, Iraq

Received on: 16/10/2012

\author{
Aida Younis Muhammad Hala Nafi Fathi \\ College of Sports Training \\ University of Mosul, Mosul, Iraq
}

\section{ABSTRACT}

Accepted on: 30/01/2013

The aim of this research is to use neural network in future forecasting field to show the of jumping competitions in international Olympics for (2016-2024).

Expert system named (AAA) is designed by using neural network in future forecasting field for period chain of data from 1984 to 2012, which represents 8 years period. The data represent the first three winners in running competition for $(100 \mathrm{~m}$., 200 m., 400 m., 100 m. Hurdles, 400 m. Hurdles, 4×100 Relay, 4×400 Relay), The prepared programs for this research has been done $\mathrm{C}++$. Then it forecast three future levels represented in $(2016,2020,2024)$, where the Olympic Cycle take place each 4 years.

Throughout the results it found that forecasting values are the best by using neural networks then other traditional methods used before.

This paper is depended on the results of athletes who take Olympic medals in women jumping events (long, triple, high and pole-vault) in 8 Olympic cycles, since Tokyo cycle (1984) till the last Olympic cycle in (2012). The cycle on (1984) was used as the beginning of study as it considered as the first Olympic cycle.

Keywords: forecasting, neural network, expert system, jumping competitions.

$$
\begin{aligned}
& \text { التنبؤ الاكتروني لفعاليات الاركاض للنساء باستخدام الشبكات العصبية } \\
& \text { هالة نافع فتحي } \\
& \text { عائدة يونس محمد } \\
& \text { فارس غانم أحمد } \\
& \text { كلية التربية الرباضية } \\
& \text { كلية طب الأسنان } \\
& \text { جامعة الموصل، الموصل، العراق لتربله } \\
& \text { جامعة الموصل، الموصل، العراق } \\
& \text { تاريخ قبول البحث: 2013/01/30 } \\
& \text { تاريخ استلام البحث: 2012/10/16 } \\
& \text { الفائزين الأوائل في مسابقات الاركاض للنساء في الأولمبياد العالمية للأعوام (2024-2016) } \\
& \text { إذ تم تصميم نظام خبير باسم (AAA) باستخدام الثبكات العصبية في مجال التتبؤ المستقبلي لبيانات } \\
& \text { سلسلة زمنية للفترة من (1984) ولغاية (2012) ولكل } 4 \text { سنوات، أي تمثل } 8 \text { فترات زمنية لبيانات تمثل الفائزين }
\end{aligned}
$$



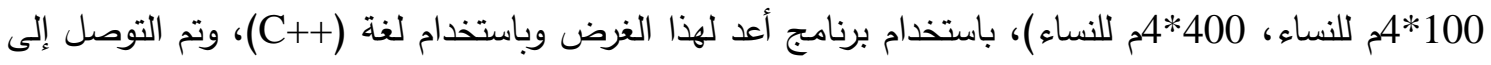

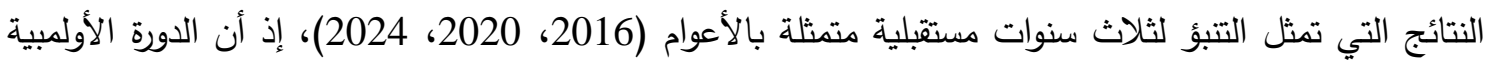

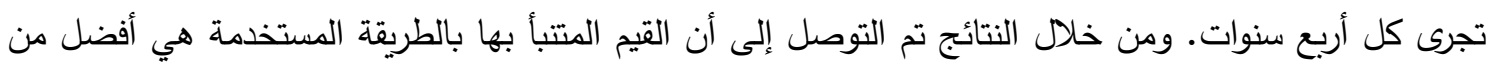

$$
\begin{aligned}
& \text { الطرائق التقليدية المستخدمة في التتبؤ سابقاً. }
\end{aligned}
$$


لقد استتد البحث على نتائج حاملي الميداليات الأولمبية في مسابقات الأركاض للنساء المذكورة في أعلاه

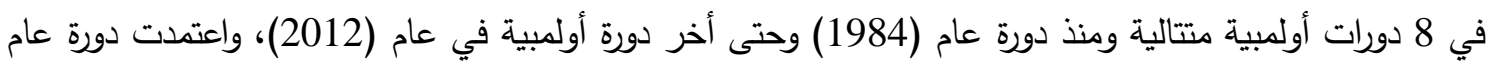
(1984) نقطة بداية السلسلة لأنها أول دوره أولمبية.

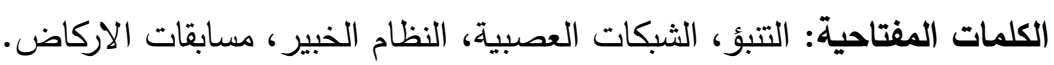

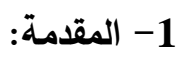

تعد عملية التتبؤ بالقيم المستقبلية من الأهداف الأساسية للعلوم الإحصائية حيث إن للتنبؤ أهمية كبيرة في عملية التخطيط واتخاذ القرارات في المجالات كافة، وعن طريق التتبؤ نستطيع التعرف على الظروف والحالاته المحيطة بالمشكلة قيد الدراسة قبل اتخاذ أي قرار. لذلك فأن موضوع التتبؤ قد لاقى ويلاقي اهتماما كبيرا ومتزايدا من قبل الباحثين وأصحاب القرارات. وقد أهتم الباحثون بتطوير الأساليب المختلفة لغرض إجراء التتبؤ، ويعد التتبؤ باستخدام الثبكات العصبية

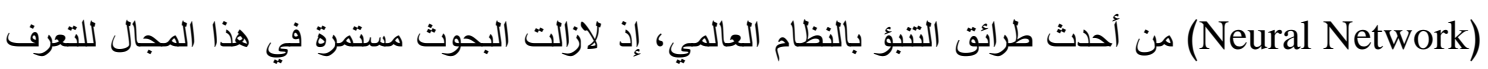
على فعالية ذلك الأسلوب. ويمكن القول أن الغاية الأساسية من طرائق السلاسل الزمنية تتمثل بالحصول على نماذج يمكن استعمالها


الملائم لبيانات السلسلة الزمنية [2]. وتكمن أهمية البحث كونه دراسة تتابعيه دقيقة مستتدة على النتائج المتحققة

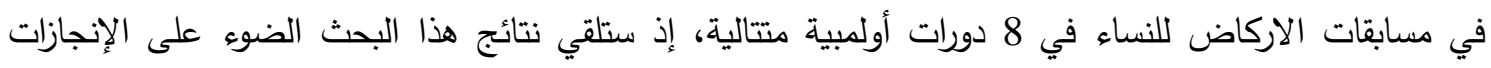
المتوقعة أي المتنبأ بها في ثلاث دورات أولمبية مستقبلية للسنوات (2024-2016) في مسابقات الأركاض (100م

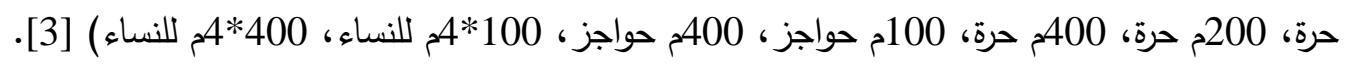

تزداد أهمية التتبؤ في السلاسل الزمنية بثكل مطرد وذلك لعلاقته الوثيقة بعجلة التطور لمختلف العلوم, وفي السنوات الأخيرة نجد أن اهتمامات الباحثين قد تزايد في تطوير أساليب التكهن وذلك باستخدام مختلف التقنيات الحديثة مثل الثبكات العصبية. وقد قدم كل من [10] بحثاً لتقليل حالة التشويش (Noise) في التكهن للسلاسل الزمنية, واستخدمت شبكات عصبية متعددة الطبقات مع إمكانية تصفية البيانات من أي ضوضاء أي تكون (Smoothing) (كما استخدمت طريقتان للمقارنة هما: التتعيم باستخدام الطرائق الإحصائية والتتعيم باستخدام خطأ التتبؤ، وتوصلا إلى أن التتبؤ بالتشويش للسلسلة الزمنية التي استخدمت الثبكات العصبية متعددة الطبقات أفضل من النموذج الذي استخدم خطأ التكهن. واستخدت الباحثة أمين بك [2] الثبكات العصبية في التكهن بسلاسل زمنية وبالتطبيق على استهلاك الطاقة الكهربائية واعتمدت على احد نماذج السلاسل الزمنية في الثبكات العصبية.

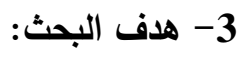

يهدف البحث إلى استخدام الثبكات العصبية في مجال التتبؤ المستقبلي، لغرض تحديد والتعرف على التى مواصفات الفائزين الأوائل في مسابقات الأركاض للنساء في الأولمبيات العالمية للسنوات (2024-2016). 


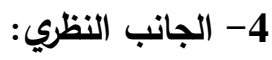

تستخدم السلاسل الزمنية بشكل واسع لدراسة تغير الظاهرة خلال فترة من الزمن كما تستخدم للتنبؤ بتغير

هذه الظاهرة في المستقبل، وذلك لسهولة إجراء الدراسة من جهة، وسهولة الحصول على على المعلومات بالكمية والنوعية المناسبة والنتائج الجيدة التي يمكن التوصل إليها عند استخدام الطرائق والمعادلات والمعلومات المناسبة فئه من جهة أخرى. فتحليل السلسلة الزمنية، هو معرفة التغيرات التي تطرأ على الظاهرة موضوع البحث خلال مدة التهات معينة وتحليل تلك التغيرات إلى أنواعها المختلفة ثم قياسها وتحديد نماذج التغير الحالية للسلسلة الزمنية وإعطاء

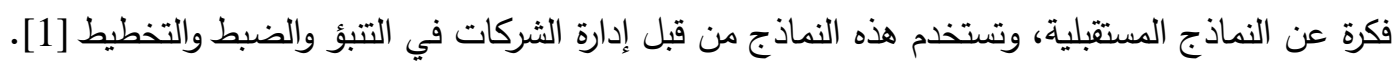

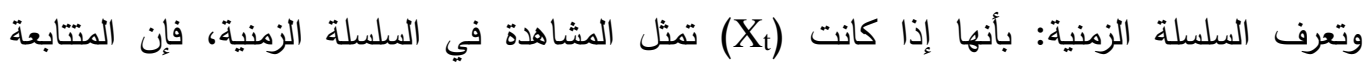
$X_{t} ; t=0, \pm 1, \pm 2$, ) (Ordered Random Variables) هن المتغيرات العشوائية المرتبة (Sequence) (111) (Multi Dimensional Probability) معرفة في فضاء احتمال متعدد الأبعاد (......

4-4 النماذج الرياضية للسلاسل الزمنية: Mathematical Models for Time Series

Autoregressive Model :1-4 نموذج الاتحدار الذاتي: 1-4

Process ) P بأنها عملية انحدار ذاتي برتبة (XR

(Autoregressive of Order P $X_{t}=a_{1} x_{t-1}+a_{2} x_{t-2}+\ldots . .+a_{p} x_{t-p}+e_{i}$

حيث أن: Autoregressive Parameters

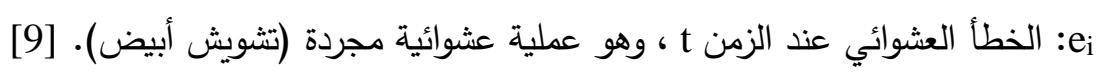

Moving Average Model : 2-4 نموذج الأوساط المتحركة

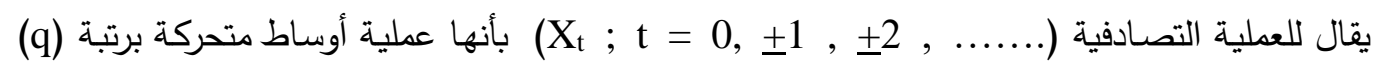

ويرمز لها بالرمز (Moving Average of Order q) $X_{t}=e_{t}+b_{1} e_{t-1}+b_{2} e_{t-2}+\ldots . .+b_{q} e_{t-q}$

Moving Parameters Average حيث أن:

[9]

1-4-4 النماذج المختلطة ذات انحدار ذاتي وأوساط متحركة: Autoregressive Moving Average Models (ARMA)

إن العناصر الأساسية لنموذج الانحدار الذاتي والأوساط المتحركة يمكن أن تدمج للحصول على تشكيلة

من النماذج تسمى نماذج انحدار ذاتي ذي أوساط متحركة برتبة (ARMA(p,q)) (p, q) وتكون بالثكل الآتي:

$X_{t}=a_{1} x_{t-1}+\ldots .+a_{p} x_{t-p}+e_{t}+b_{1} e_{t-1}+\ldots .+b_{q} e_{t-q}$

Akaike's Information Criterion :4-4-4 معيار أكايكي للمعلومات

ظهـرت في بدايـة السـبعينات بعض المعـيير لتثـخيص النمـوذج المطلـوب دون الاعتمـاد على دوال الارتباط، فقد أقترح العالم الياباني (Akaike) معياراً عامـاً في تطوير ونمذجـة السلاسل الزمنية يدعى (AIC) 



كما يمكن استخدامه في مجالات إحصائية أخرى. وتحسب قيمة (AIC) كما يلي: $A I C k=n \operatorname{In}\left(\sigma_{\varepsilon}^{2}\right)+2 k$

$$
\begin{aligned}
& \text { حيث أن: n مجم العينة. } \\
& \text { Residuals Variance تباين البواقيه }
\end{aligned}
$$$$
\text { ع عدد معلمات النموذج المقدرة. }
$$

4-2-4 الثبكات العصبية الاصطناعية: Artificial Neural Networks (ANN)

تعنى الثبكة العصبية بحقل تقنية المعلومات، وهي نظام من البرامج وهياكل البيانات التي تقارب عمل

الاماغ البشري، فنتضمن الشبكة العصبية عدداً كبيراً من المعالجات التي تعمل على التوازي. تبدأ الحالة الابتدائية للثبكة العصبية بعملية "التدريب" أو تلقي كميات من البيانات والقواعد عن العلاقات بين تلك البيانات ثم يوجهها

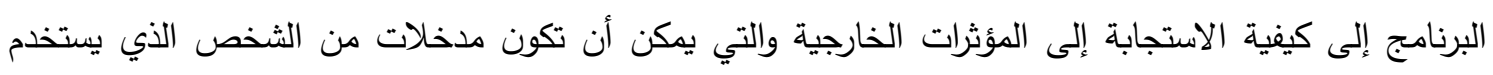

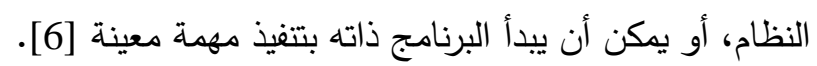

إن المفتاح لأنموذج الثبكة العصبية الاصطناعية، هو الهيكل لنظام معالج البيانات الذي يقوم بربط ديطة

وتتظيم عدد كبير من عناصر المعالجة المرتبطة داخلياً (العصبونات) والتي تعمل بانسجام، وتمر الإثارات بين العقد (العصبونات) عبر خطوط ربط ويرفق كل خط بوزن معين، وتضرب الإشارات الداخلة إلى العقدة (العصبونة) بهذه الأوزان وتجمع المدخلات الموزونة في العقد أو العصبونات وبعد ذلك يتم معالجة مخرجات كل على عقدة بواسطة

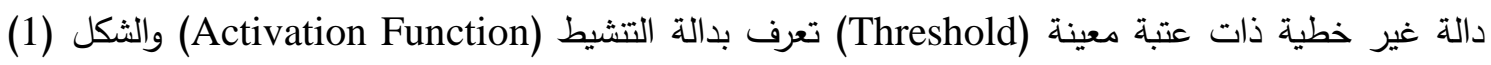
يوضح شبكة عصبية اصطناعية بسيطة [5].

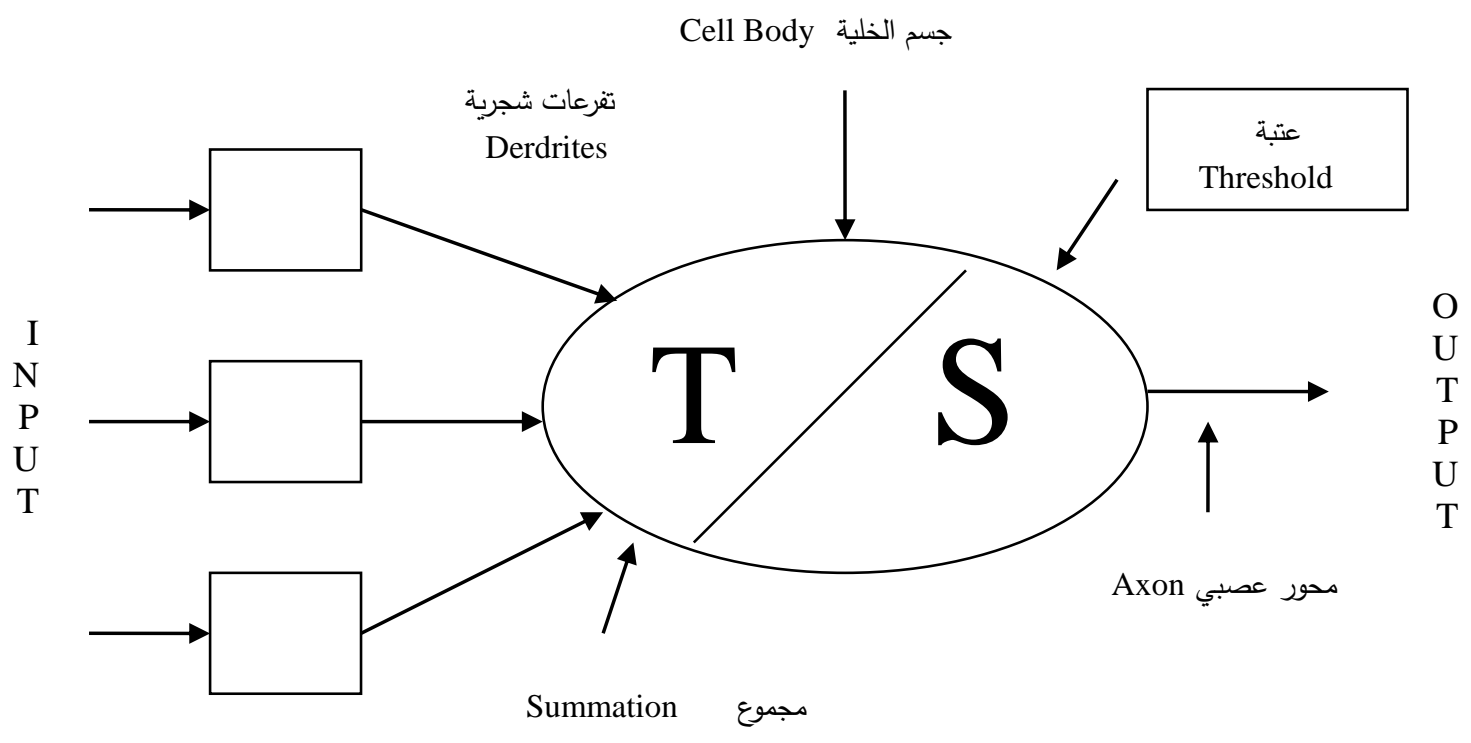

Hidden layers

شكل (1). مخطط عمل الثبكة العصبية الاصطناعية 
• إن الثبكات العصبية أعطت حلولاً ذات كفاءة عالية للكثير من التطبيقات في العديد من المجالات منها:

$$
\text { • القدييز الأنماط والتعرف على الصور • التحى التعرف على الصور المشوهة. }
$$

إكمال الصور التي فقدت جزء منها، مثل الصور المرسلة بواسطة الأقمار الصناعية.

عمليات التصنيف إلى عدد من الفئات

4-2-1-1 الثبكات العصبية ذوات الانتشار العكسي: Back Propagation Neural Networks

إن شبكات التغذية الخلفية أو العكسية يمكنها أن تمتلك إثارات تتحرك أو تتتقل في كلا الاتجاهين

بواسطة دارات في الثبكة. وهي قوية ومعقدة جداً، وهي شبكات حركية يتغير استقرارها باستمرار إلى حين الوصول

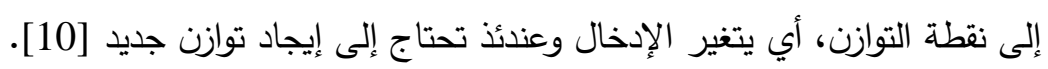

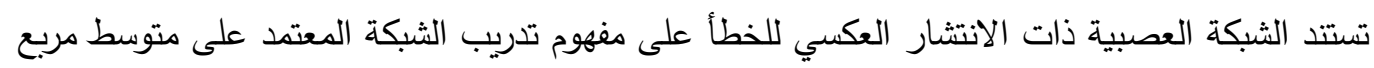

الخطأ، وذلك بإيجاد القيمة الصغرى لمربع الخطأ لقيمة المخرجات المحسوبة من قبل الثبكة، حيث تُحدَّث الأوزان بين الطبقات لحين الوصول إلى الأوزان المثلى التي تعطي أفضل توفيق للنموذج. إن الانتشار العكسي للخطأ

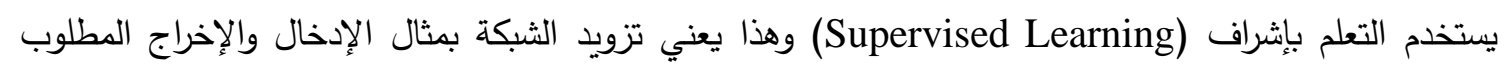

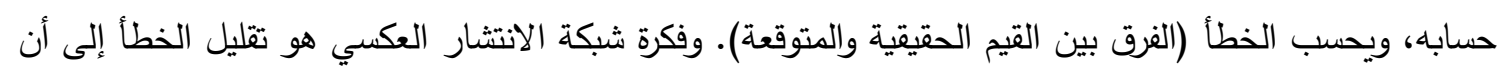

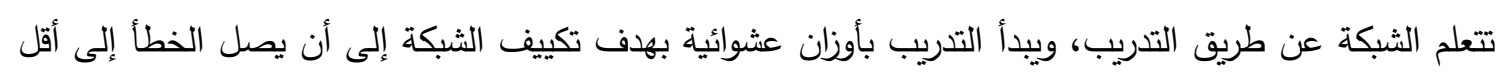
ما يمكن. [7]

\section{4-2-2-2 خوارزمية الثبكة العصبية ذات الاتتشار العكسي:}

إن خوارزمية شبكة الانتشار العكسي للخطأ من أهم خوارزميات شبكات التدريب وتعتمد على اختيار دالة

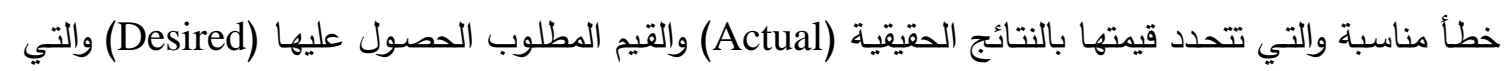
$\mu=\sum_{i=1}^{N} w_{i} x_{i}$ تعتمد أيضاً على معالم الثبكة مثل الأوزان والعتبة: $Y=f(\mu-\theta)$

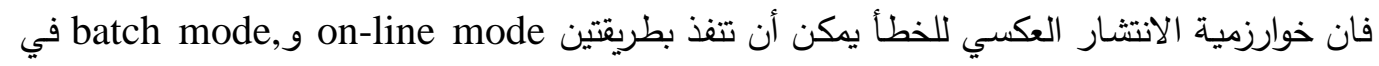
نموذج on-line فان دالة الخطأ تحسب بعد كل نموذج إدخال وإشارة الخطأ تبث رجوعا خلال الثبكة وتعدل 
الأوزان ودالة الخطأ الدحسوبة هي متوسطة مربعات الخطأ (Mean Square Error (MSE) بين القيم الحقيقية والقيم المرغوب بها، والأوزان الجديدة تستخدم في حساب النموذج. وتمثل جميع النماذج يسمى التكرار Iteration.


يقيم إدخال القيم جميعاً، ودالة الخطأ تحسب مجموعة الخطأ (MSE) لكل الإشارات وجميع النماذج قبل تمثيل النموذج التالي، مما يعني أن هذا النموذج يقوم بحساب (MSE) لكل وحدات المخرجات أو لجميع النماذج. [8] 4-4 التنبؤ باستخدام الثبكات العصبية:

يعد التتبؤ باستخدام الثبكات العصبية من الأساليب الحديثة التي لاقت اهتماما واسعاً في مجالات متعددة

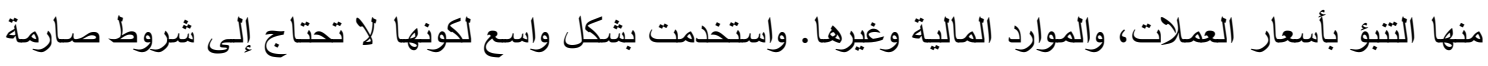

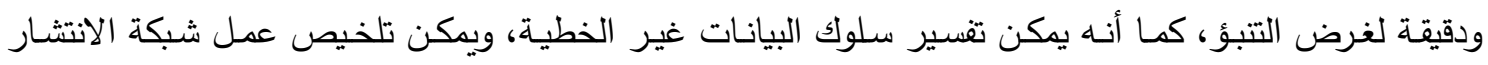

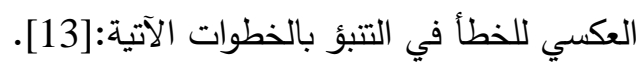

الخطوة الأولى: اختيار المتغيرات Variables Selection : إذ يجب اختيار المشاهدات للمتغيرات بحيث تمثل باتل المشكلة تمثيلاً جيداً.

الخطوة الثانية: معالجة البيانات Data Processing: إجراء بعض العمليات على البيانات المستخدمة مثل تحديد الاتجاه العام، التركيز على العلاقات بين المشاهدات، إيجاد توزيع البيانات.

الخطوة الثالثة: تقسيم البيانات إلى المجاميع Divide Data Into Sets: تقسم البيانات المتوفرة إلى مجاميع تلئي التالية:

1) مجموعة التدريب Training Set: مجموعة تعلم وتحديد نموذج للبيانات.

2) مجموعة الاختبار Testing Set: والتي يمكن عن طريقها تقرير مهارة الثبكة الافتراضية

$$
\text { وإمكانية استخدامها بصورة عامة. }
$$

3) مجموعة الثرعية Valid Action Set: وهي مجموعة لإجراء اختبار نهائي لأداء الثبكة. الخطوة الرابعة: نموذج (مثال) الثبكة العصبية Neural Network Paradigms: عند تحديد نموذج الشبكة

$$
\text { العصبية يجب اختيار الآتي: }
$$

• • عدد العصبونات للإدخال والذي يساوي عدد المتغيرات المستقلة.

عدد الطبقات المخفية والذي يعتمد على قيمة الخطأ المستخدم في الثبكة.

$$
\text { • • • ع عدد العصبونات المخفية والذي يحدد عن طريق التجربة. }
$$

الخطوة الخامسة: معيار التقييم Evaluation Criteria: إن معيار المستخدم في شبكة الانتثار العكسي لتقييم

الخطأ هو مجموع مربعات الأخطاء Mean Square Error (MSE).

الخطوة السادسة: تدريب الثبكة Neural Network Training وتضم هذه الخطوة:

• تعليم النموذج: إيجاد مجموعة الأوزان بين العصبونات والتي تحدد أقل قيمة لمربع الخطأ.

• خوارزمية شبكة الانتشار الخلفي للخطأ: تستخدم خوارزمية التدريب لتقليل الميل.

الخطوة السابعة: التتفيذ Implementation: وهي من أهم الخطوات حيث تختبر الثبكة من حيث قدرة التكيف مع حالة التغير في دورة وإمكانية إعادة التدريب والوصول إلى أقل مربع خطأ عند تغير البيانات، 
ويتم بناء الثبكات العصبية عن طريق البرمجة (أي أن الثبكة العصبونية هي عبارة عن

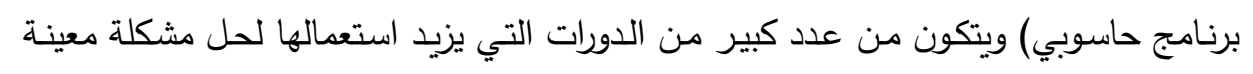
[2]. ويوضح الثكل رقم (2) خوارزمية تدريب الثبكة ذات الانتشار العكسي للخطأ.


شكل (2). خوارزمية تدريب شبكة الانتشار العكسي

من الأمور التي تثجع على استخدام الثبكات العصبية في معالجة السلسلة الزمنية التي نحن بصدها هو

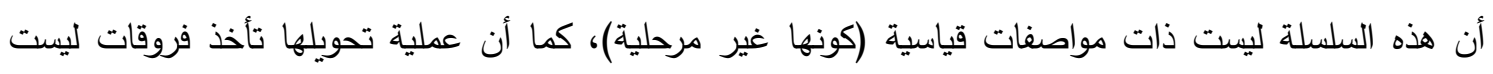


لقد تم تصميم برنامج حاسوبي بعنوان (AAA) للحصول على قيمة التتبؤ للسلسلة الزمنية للمشاهدات




حيث أن المدخلات هي نتائج حاملي الميداليات الأولمبية في مسابقات الأركاض (100م حرة، 200م حرة، 400م

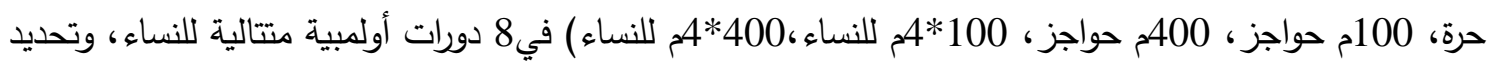
عدد العقد المخفية والذي يتم من خلال التدريب، إذ يتضمن إجراء العديد من التجارب الحاسوبية. وكذلك يمكن

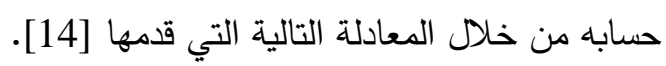

$$
N_{\text {hidden }} \leq \frac{N_{\text {train }} E_{\text {tolernce }}}{N_{p s t}+N_{\text {output }}}
$$

Nhidden

N N $N_{\text {train }}$

Etolerance

N N : ميثل عدد البيانات التي تم إجراء التدريب عليها.

Noutput

$N_{\text {train }}=$ Etolerance كي يكون (0.01) وعدد المرات التدريب البحث تم تتبيت مقدار الخطات

$$
\begin{array}{rl}
N_{\text {output }}=1 & 200 N_{p s t} \\
N_{\text {hidden }} \leq & \frac{2 N_{p t s}}{N_{p t s}+1}
\end{array}
$$

وتم اختيار عدة نماذج من البيانات وتبين أن النموذج الملائم للبيانات ولجميع (الألعاب) هو (ARIMA) وتم تصميم برنامج بلغة (C++) وباستخدام الثبكات العصبية للنموذج (ARIMA)، تم فحص السلسلة وتحويلها من حالة عدم الاستقرار إلى حالة الاستقرار وجعلها مستقرة حول الوسط الحسابي والتباين ومن ثم رسم سلسلة

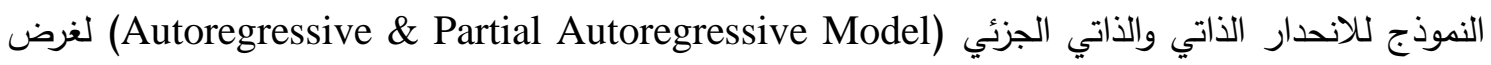
اختيار النموذج الملائم، ومن ثم تم أخذ عدة احتمالات للنموذج المنتخب من درجات مختلفة وبدرجات إزاحة

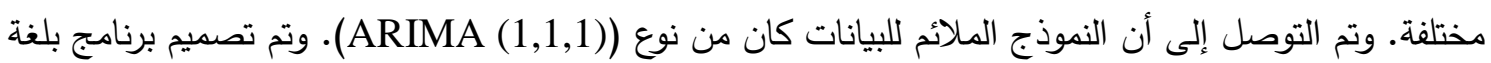
C++ واستخدام تكنيك الثبكات العصبية لغرض التتبؤ بالنتائج واعتماداً على البيانات الدذكورة سابقاً. أما لاختيار عدد العصبونات المخفية، فقد استخدمت جميع المشاهدات وأجري الاختيار باستخدام شبكة باتئي الانتشار العكسي للخطأ المعياري أكايكي (AIC) وتم تدريب الثبكة باستخدام عدة عصبونات مخفية أفضلها كان عددها (7) والجدول (1) يوضح مقدار الخطأ المعياري المطلق (AIC) للمسابقات الأولمبية للأوائل الثلاثة في

\begin{tabular}{|c|c|c|c|c|}
\hline عدد العصبونات & $\begin{array}{c}\text { القيمة المطلقة } \\
\text { AIC } 3\end{array}$ & $\begin{array}{c}\text { القيمة المطلقة } \\
\text { AIC } 2\end{array}$ & $\begin{array}{c}\text { القيمة المطلقة } \\
\text { AIC1 }\end{array}$ & الألعاب \\
\hline 7 & 0.0212 & 0.2361 & 0.4121 & $100 \mathrm{~m}$ \\
\hline 7 & 0.0206 & 0.2214 & 0.0203 & $200 \mathrm{~m}$ \\
\hline 7 & 0.1931 & 0.2041 & 0.0190 & $400 \mathrm{~m}$ \\
\hline 7 & 0.2092 & 0.2072 & 0.2311 & 100 Hurries \\
\hline 7 & 0.1972 & 0.0280 & 0.2063 & 200 Hurries \\
\hline 7 & 0.1041 & 0.0202 & 0.0201 & $4 * 100$ Relay \\
\hline
\end{tabular}
الأركاض للنساء.

الجدول (1). اختيار عدد العصبونات المخفية 


\section{\begin{tabular}{l||l||l}
\hline 7 & 0.1003 & 0.0221
\end{tabular}}

0.1592

4*400 Relay

في هذه الاختبارات تم تثبيت عدد مرات التدريب باعتمادها (200) تكرار وقيمة مربع الخطأ (MSE=0.01) وكانت الأوزان (0.55) وبذلك عد أفضل عدد للعصبونات هو (7) في مسابقات الأركاض للنساء، وذلك لأن القيمة المطلقة AIC في هذه الحالات هي الأقل. وفي هذا الاختبار استخدمت شبكة عصبية ذات الانتشار العكسي للخطأ بعدد عصبونات (7) على لونى

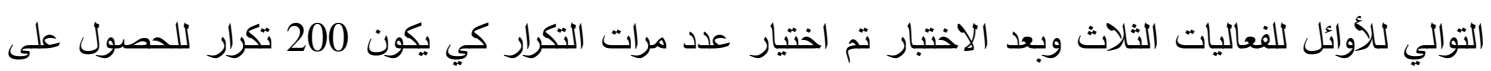
أقل قيمة إكايكي AIC. وقد تبين أن أفضل شبكة (Best Network) عند (200) تكرار . ولغرض التطبيق العملي للشبكة العصبية ذات الانتشار العكسي للخطأ فقد استخدام برنامج حاسوبي مصمم بلغة القيمة المطلقة Cل للتوصل إلى قيم التتبؤ وحساب المعلومات الإحصائية وتم بناء شبكة عصبية ذات التئية انتشار عكسي للخطأ للمشاهدات الأصلية وباستخدام العقد المخفية (Hidden Nodes) لكل مسابقة (7)، وكما موضح في الجدول أعلاه، وطبقتين مخفيتين (Hidden Layers Nodes) حيث يبدأ التدريب بأوزان عشوائية ثم $X O i=\operatorname{seg}\left(X i+I^{*} W j\right)$ تطبيق الصيخ الخاصة بالانتقال الأمامي الآتي: ملنين

$$
\begin{aligned}
& X O i=\operatorname{seg}(X i+I * W j) \quad \ldots(8) \\
& \text { حيث أن: } \\
& \text { XOi } \\
& \text { X } \\
& \text { I } \\
& \text { W : الأوزان الابتدائية. } \\
& \text { ولحساب مخرجات الطبقات المخفية تستخدم الصيغة: } \\
& \left.O L(J)=\operatorname{seg} \mid \sum_{i=o}^{N} \times O[i] * W[J][i]\right\rfloor \\
& \text { حيث أن: } \\
& \text { J : الطبقة المخفية في هذا التطبيق. } \\
& O 1=\operatorname{seg}\left[\sum_{1=1}^{7} O L(J, i) * W O i\right] \\
& \text { N : عدد المشاهدات. وتحسب في طبقة الإخراج وفق الصيغة: } \\
& W O_{i}=n * Z * O_{L}(n, i)
\end{aligned}
$$




$$
\text { O }
$$

Sigmoid ما يكافئ مشتقة دالة. Seg D

$e=|o d-o|$

$O l_{2, i}=Z * W O_{I} * \operatorname{seg} D\left(o L_{2, i}\right)$.

أما الانتقال الخلفي فيحسب وفق الصيخ التالية:

$W_{i, j}=W i j+\eta * O L_{1, i} * O l_{2, i}$

$O l_{1, i}=\sum_{J=1}^{2} O L_{2, j} * W_{i, j} * \operatorname{seg} D\left(O L_{1, i}\right)$

$W I i=w I i+\eta * O L_{1-i}$.

$$
E=e / \sum I
$$

ويحسب الخطأ الكلي للثبكة كما يلي:

ويستمر التدريب للشبكة لحين الوصول إلى قيمة الخطأ المحدد للثبكة (0.01)، ومن خلال تدريب الثبكة

العصبية تم التوصل إلى قيم التتبؤ وحساب المعلومات الإحصائية. والثكل (3) يوضح نتائج الثبكة العصبية والأوزان الناجحة من مسابقات الاركاض للنساء الثلاثة الأوائل.

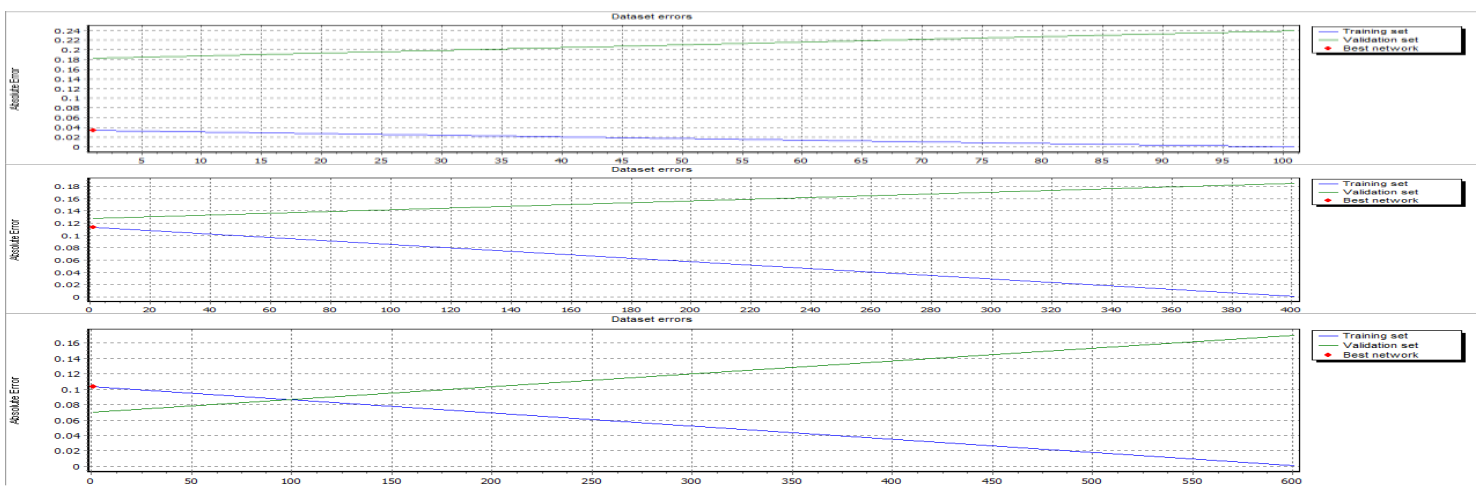

فعالية (100M)






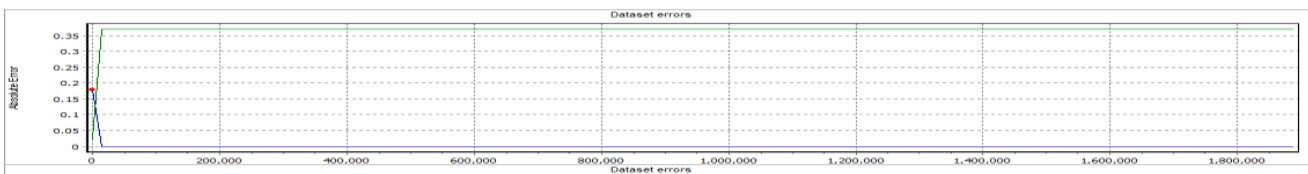

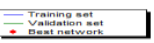


Frater

فعالية (400M)
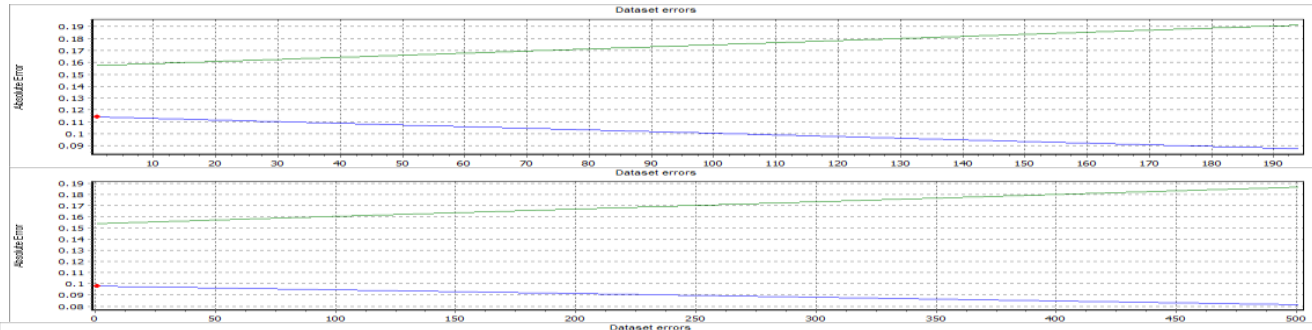

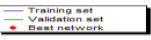

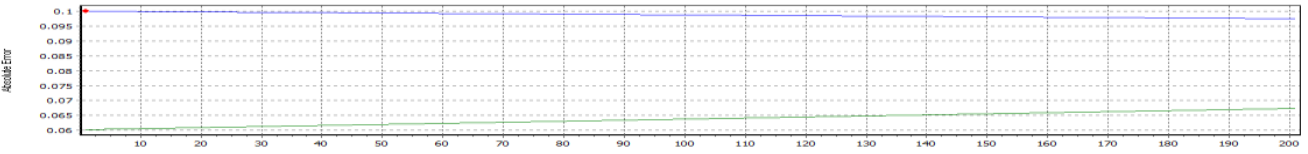

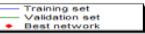

فعالية 100m Hurdles

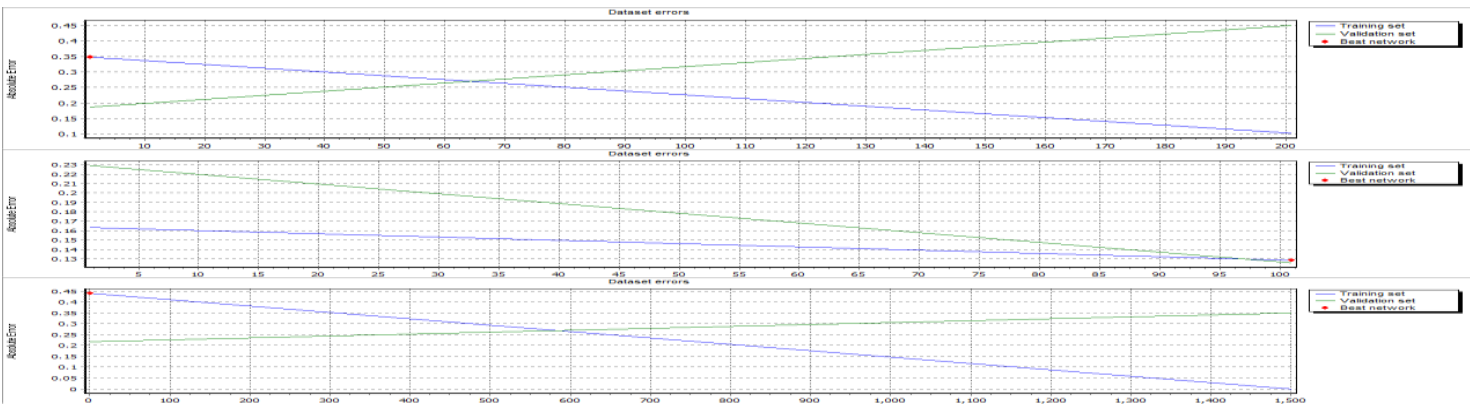

فعالية 400m Hurdles


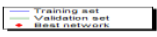

فعالية 40100 Relay

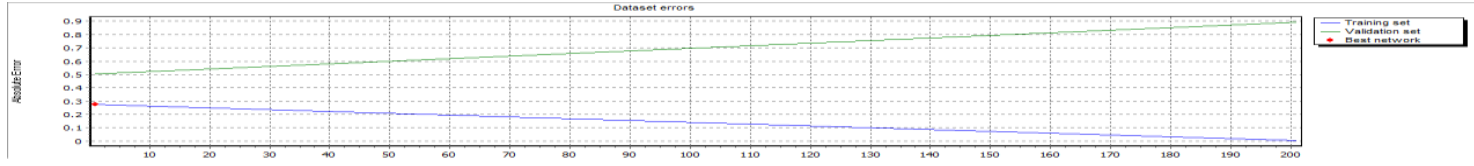




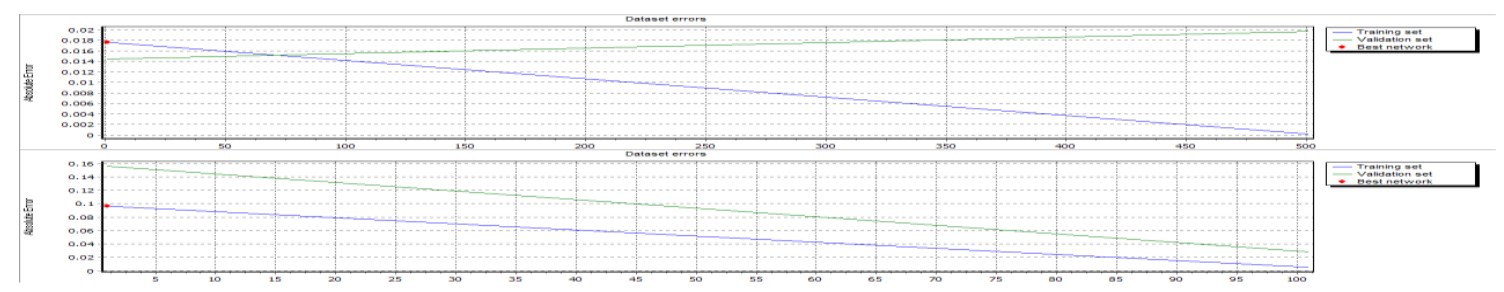

\section{فعالية 400 Relay}

الثكل (3). نتائج الشبكة العصبية ذات الانتشار العكسي للخطأ

وبعد إتمام عمليات تدريب الثبكة العصبية لنتائج مسابقات الاركاض للنساء في الدورات الأولمبية

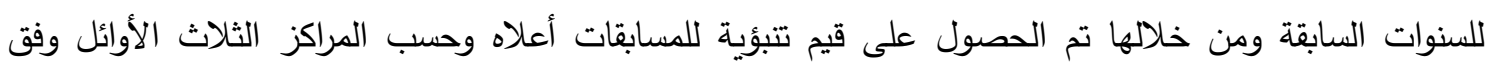

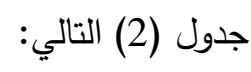

جدول (2). يبين القيم التتبؤية للمسابقات حسب المراكز في الثبكات العصبية

\begin{tabular}{|c|c|c|c|c|c|c|c|c|}
\hline $\begin{array}{l}4400 \\
\text { Reloy }\end{array}$ & $\begin{array}{l}* 100 \\
\text { Reloy }\end{array}$ & $\begin{array}{c}200 \\
\text { Hurties }\end{array}$ & $\begin{array}{c}100 \\
\text { Hurties }\end{array}$ & $400 \mathrm{~m}$ & $200 \mathrm{~m}$ & $100 \mathrm{~m}$ & السنوات & المراكز \\
\hline 3.17 & 41.99 & ב50.02 & 12.37 & 488.98 & 22.1 & 10.77 & 2016 & \multirow{3}{*}{ الأول } \\
\hline 3.18 & 41.99 & 52.64 & 12.54 & 48.98 & 22.5 & 10.93 & 2020 & \\
\hline 3.19 & 41.99 & 52.85 & 12.58 & 48.98 & 21.92 & 10.95 & 2024 & \\
\hline 3.22 & 42.61 & 53.08 & 12.37 & 49.70 & 22.3 & 10.92 & 2016 & \multirow{3}{*}{ الثاني } \\
\hline 3,23 & 42.60 & 53.70 & 12.46 & 49.46 & 22.4 & 10.98 & 2020 & \\
\hline 3.29 & 42.55 & 53.77 & 12.95 & 49.89 & 22.10 & 10.99 & 2024 & \\
\hline 3.33 & 42.70 & 53.22 & 12.48 & 49.63 & 22.17 & 11.6 & 2016 & \multirow{3}{*}{ الثالث } \\
\hline 3.33 & 42.75 & 53.44 & 12.64 & 49.64 & 22.17 & 11.5 & 2020 & \\
\hline 3.34 & 42.72 & 53.48 & 12.76 & 49.65 & 22.17 & 11.1 & 2024 & \\
\hline
\end{tabular}

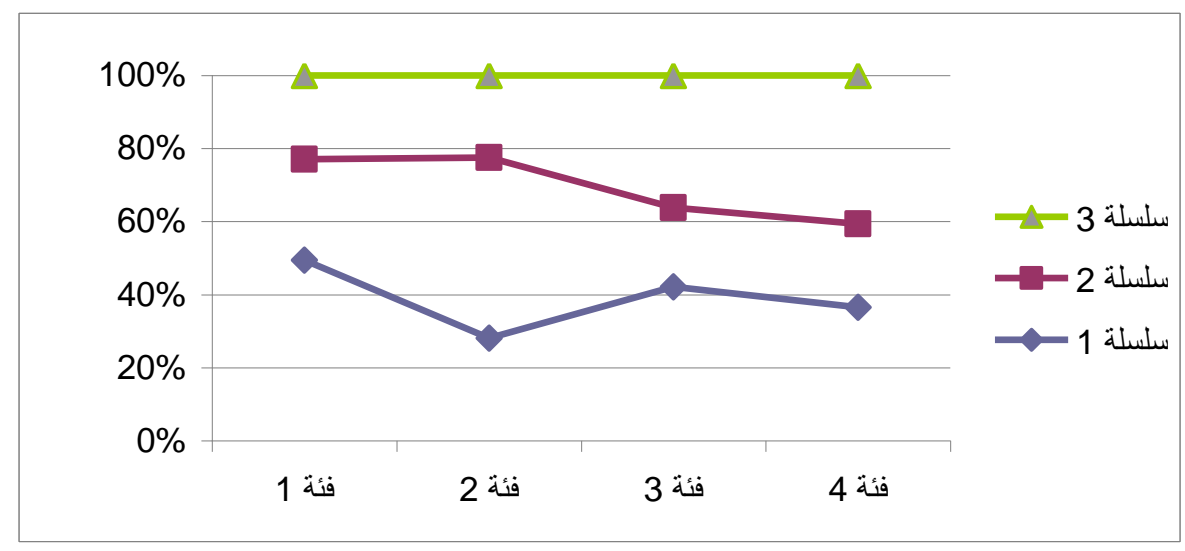

شكل (4). تبين نتائج القيم التتبؤية للمسابقات حسب المراكز في الثبكات العصبية.

الاستنتاجات:

إن أهم الاستتتاجات التي تم التوصل إليها من خلال هذه الدراسة هي تفوق الثبكات العصبية على

الطرائق الكلاسيكية حيث تم الحصول على نتائج ذوات قيم أقل للمعايير الإحصائية المستخدمة لحساب خطأ التتبؤ، لذلك تعد الثبكات العصبية الاصطناعية هي الطريقة الأفضل والأكثر دقة للتتبؤ بالقيم المستقبلية للسلسلة الزمنية قيد الدراسة، مما يشجع استخدام هذا الأسلوب في التتبؤ بالسلاسل الزمنية المختلفة. 


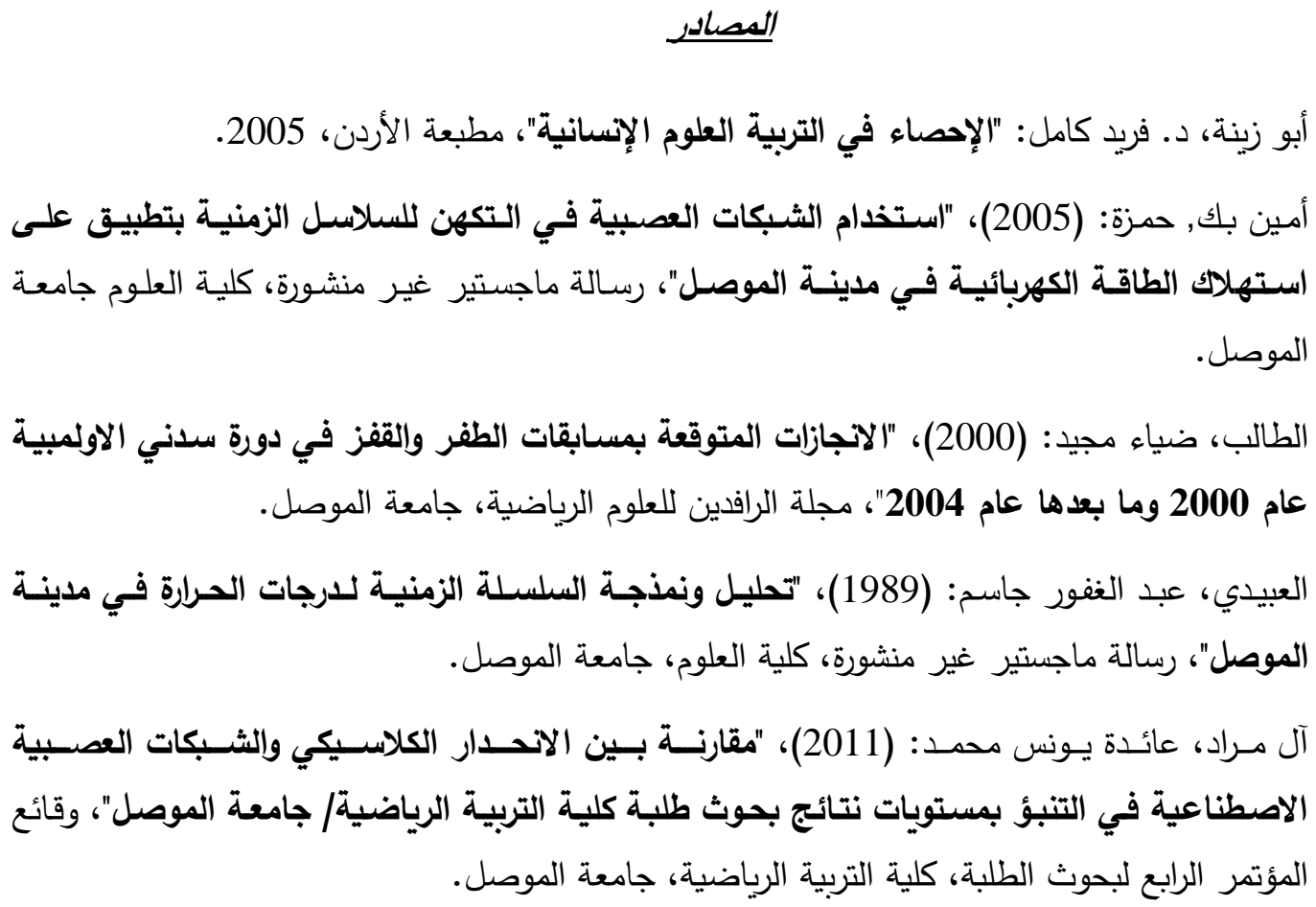

Http://www.(4arab.com.mejhar_php.(2011) الموسوعة العربية للكمبيوتر والانترنيت.

الناصر ، عبد المجيد حمزة والعبيدي، مروان عبد الحميد، (2003)، "استخدام الثبكات العصبية للتتبؤ

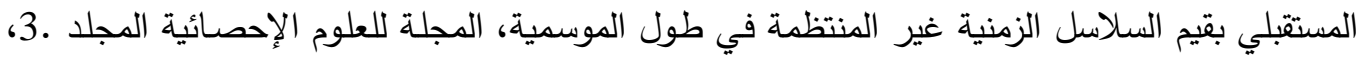

$$
\text { العدد 6، كلية علوم الحاسبات والرياضيات، جامعة الموصل. }
$$

[8] Ampazis, N. (1998). "Introduction to Neural Networks", www.iitnrcps.ariadneet.grlnearal/index

[9] Makridakis, Spyros; Wheel Wright, Steven C., and Hyndman, Rob J.d. So, (1998), " FORECASTING Method and Applications", $3^{\text {rd }}$ ed., John Wiley n, Inc., USA.

[10] Ostrich U. and dobnikar A., (2001), "wave based smoothing in time series prediction with normal networks", university of Ljubljana faculty of computer and information science Slovenia.

[11] Rao, V. B. and Rao, H.V. (1993), "C++ Networks and Fuzzy Logie", MISPress.

[12] Smith, Leslie (2003), "An Introduction to Neural Networks", Department of Computing and Mathematics, university of Stir Ling. UK.

[13] Sinha, H. (2002) "Designing a Neurad Network for forecasting Financial and Econ omic time Series", June $4^{\text {th }}$, INDE593.

[14] Lin, Feng; Yu, Xing Huo; Gregor, Shirelyand lrons, Richard, (1995), "Time Series Forecasting wi NetworksInternational, Volume 02, IssN 1320-0682, Australia. 